\title{
Crohn's Disease: A Curable Disease Held Hostage?
}

\section{Gilles RG Monif*}

Infectious Diseases Incorporated, University of Bellevue USA

*Corresponding author: Gilles RG Monif, Infectious Diseases Incorporated, University of Bellevue, NE, USA.
Received Date: May 15, 2021

Published Date: May 28, 2021

\begin{abstract}
Introduction
Permanent remissions have been attained in Crohn's disease. Nevertheless, the current focus of therapy remains sclerosed with the prevailing drug therapy through which temporary remissions are attainable. FDA's reliance on evidence-based validation through comparative, placebo controlled, double-blinded studies has created an impediment to acceptance of divergent alternated therapies as being within the standard of care. In so doing, FDA has basically surrendered the therapeutic dialogue of Crohn's disease to those who can pay for such studies.
\end{abstract}

Keywords: Crohn's disease; Hruska Postulate; Permanent remission

\section{Introduction}

The current standard of care of Crohn's disease evolved from trial-and-error therapeutic attempts to address the signs and symptoms of disease. The prevailing standard of care is based on "evidence-based" data, demonstrating that the current drugs of choice produce a statistically significant improvement of disease over placebo or alternative therapy. For over two decades, disruption of the effector arm of the immune response has been the foundation of Crohn's disease therapy. Despite not having produced cures in over a decade of clinical use, biologics have attained the status of drugs-of-choice for Crohn's disease. The rush to disease plication has overshadowed the clinical observations that two divergent therapeutic approaches (diet and anti-tuberculosis drugs) have produced occasional permanent remissions. These isolated instances of permanent remission induction have been glossed over by their failure to constitute being "evidence-based" data. For the Food and Drug Administration (FDA) and other government agencies to accept a clinical therapeutic outcome, the data needs to be "evidence-based" derived from primarily comparative, doubleblinded, placebo-controlled studies.

In 2015, a pathogenesis for Crohn's disease was introduced that put into focus the shared mechanism by which dietary manipulation and selected anti-mycobacterial therapy had achieved long-term remissions/cures [1]. The Hruska Postulate proposed the thesis that, in the absence of functional acquired immunity, a newborn infected by Mycobacterium avium subspecies paratuberculosis (MAP) could be so challenged to abort mycobacterium replication that its inherent immune system became fixed within immunological memory. Owing to the loss of immunological tolerance, every time the baby's immune system encountered MAP. it would again respond by elaborating pro-inflammatory cytokines directed at MAP at its points of mucosal attachment to the gastrointestinal mucosa and its antigen processing. A major factor complicating attempts to construct meaningful comparative therapeutic groups for clinical trials is the degree to which the gastrointestinal microbiota has 
altered gastrointestinal structure and function [2-4]. Once mucosal integrity is lost, the microbiota of the gastrointestinal tract becomes a superimposed disease process. The failure to address the resultant polymicrobial infection is responsible for the permanent sequel of Crohn's disease: strictures, bowel-to-bowel fistulae, perianal fistula bowel perforation, sepsis, etc. Once in place, the resultant alterations of structure and function create therapeutic subgroups which necessitated added therapy. Given biologics and steroids failure to attain but temporary remissions, the under-addressed question is how do diet or selected anti-microbial drugs induce isolate cures?

That diet could be a therapeutic modality has long been inferred. Before the introduction of biologics, diet had long been used in front line therapy for Crohn's disease in Japan. In the United States. selected pediatric gastroenterologists using dietary manipulations have documented a beneficial effect, but not cures [5-7]. In isolated instances, individuals afflicted with Crohn's disease have achieved permanent remission by rigid adherence to what are variations of basically vegetarian-like regimens. Chiba, et al. reported that $94 \%$ of Crohn's afflicted individuals who remained on a semi-vegetarian diet maintained their clinical remission whereas 33\% who returned to a regular diet relapsed [8] Much of this clinical response has bene attributed to reducing the volume of antigen challenges by exclusion of foods potentially adulterated by MAP [2]. The question left unanswered had been why. A veterinary experiment-in-nature demonstrated that dietary targeting to enhance host immunity produced the mass destruction of MAP with terminal Johne's disease (due to the replication of MAP) [9]. In Crohn's disease, the diarrhea and loss of absorption areas cause significant loss of key vitamins and minerals vital to the integrity of host immunity [10]. The cures achieved through dietary manipulation, reduction of antigen challenges, anabolic diet enhancement and enhancement of host immunity speak to a potential mechanism by which individuals have achieved self-cure without pharmaceutical intervention [11].

The explanation as to why selected anti-mycobacterial drugs have achieved permanent remissions is less obvious [12-14]. Unlike Johne's disease in domestic animals, Crohn's disease is not due to organism replication. MAP can't be identified with special stains nor isolated from disease tissue. Prior attempts to cure Crohn's disease using anti-tuberculosis drugs largely failed. Why selected anti-MAP therapy has produced individual cures is due to their ability, not to destroy MAP as such, but to destroy MAP that exists in its cell-wall free state. The MAPs that drive the dysfunctional immune response exist as cell wall free versions of itself. The prior failures of prior anti-tuberculosis drugs are largely attributable to their mechanism of action being directed against mycobacterium cell wall. The expanded Hruska Postulate states abortion of the immune-mediated aspect of Crohn's disease is contingent on the destruction of the sustaining MAP immune template. To destroy
MAP in its cell wall-free form, drug therapy must be able to disrupt MAP's RNA metabolism.

Given the number of years required to assess outcome, the subset of patient groups based upon degree of documented tissue damage required, age, immune status, diet, etc., make conducting a comparative, placebo-controlled, double blinded comparative study against a given biologic a near impossibility that no one will pay for. Unless FDA begins to accept small clinical trials, whose endpoint is either failure or success without continued drug therapy over a prolonged period of time to be "evidence-based", the therapy of Crohn's disease will continue to reside with those that can pay the bill for the creation of "evidenced-based" data. Without FDA validation, physicians attempting innovative therapies face loss of liability protection afforded by the "standard of care". Currently, the will to cure Crohn's disease appears subordinate to FDA's inflexibility and the elephant in the room. Chronic diseases function as a revenue enhancer for both healthcare provider and the pharmaceutical industry. In 2018, the maker of the principal biologic used in the therapy of Crohn's disease made a reputed 19.94 billion dollars. The 2018 American College of Gastroenterology's Clinical Guideline states "Despite the recent advances in the treatment of patients with $C D$, there still remains a large group of patients who do not respond adequately to our current medication armamentarium." ....... We will certainly expand our medical treatment war chest and uncover effective biologics with different mechanisms of action to treat our patient. If the initial biologic drug fails, the patient will be able to be switch to another agent and even combination biologics may become a reality [15]. The Guideline further states that the standard of care is "rigid' and "rarely violated".

\section{Acknowledgement}

None.

\section{Conflict of Interest}

No conflict of interest.

\section{References}

1. Monif GRG (2015) The Hruska postulate of Crohn's disease. Med Hypothesis 85: 878-881.

2. Monif GRG (2017) An infectious disease process within an immunemediated disease process: Role of the gastrointestinal microbiota in Crohn's disease. Adv Res Gastroenterol Hepatol 5(5): 1-2.

3. Monif GRG (2018) Are the permanent sequelae of Crohn's disease a failure to treat the gastrointestinal microbiota. Med Hypothesis 122 198-199.

4. Monif GRG (2016) Translation of hypothesis to therapy in Crohn's disease. Inflam Bowel Dis 22(2): E8-E9.

5. David L Suskind, Ghassan Wahbeh, Stanley A Cohen, Christopher Damman, Jani Klein, et al. (2016) Patients perceive clinical benefit with specific carbohydrate diet for inflammatory bowel disease. Dig Dis Sci 61: 3255-3260.

6. Stanley A Cohen, Benjamin D Gold, Salvatore Oliva, Jeffery Lewis, Angela Stallworth, et al. (2014) Clinical and mucosal improvement with specific 
carbohydrate diet in pediatric Crohn's disease. J Pediatr Gastroenterol 59: 516-521.

7. Burgis JC, Nguten K, Park KT, Cox K (2016) Response to strict and liberalized specific carbohydrate diet in Pediatric Crohn's disease. World J Gastroenterol 22: 2111-2117.

8. Chiba M, Abe T, Tsuda H (2014) Life-style-related diseases in Crohn's disease: relapse prevention with semi-vegetarian diet. World J Gastroenterol 16: 2484-2495.

9. Monif GRG (2015) Relationship of eosinophilia and acid-fast bacilli in Johne's disease. Intern J Appl Res Vet Med 13: 147-149.

10. Khabbaz R (2017) The enhanced role of diet in Crohn's disease. Adv Res Gastroenterol Hepatol 4: 1-2.

11. Behr MA, Hanley J (2008) Anti-mycobacterial therapy for Crohn's disease: a reanalysis. Lancet Infect Dis 8: 344.
12. Chamberlin W, Borody T, Campell J (2011) Primary treatment of Crohn's disease: combined antibiotics taking center stage. Expert Rev Clin Immunol 76: 751-760.

13. Biroulet IP, Neut C, Colombel JF (2008) Anti-mycobacterium therapy in Crohn's disease: the end of a long controversy. Prac Gastroenterol 1:1117.

14. Lipton JE (2012) My personal MAP-quest. The Paratuberculosis Newsletter pp. 17-18.

15. Lichenstein GR, Loftus EV, Isaacs KL (2018) ACCG Clinical Guidelines: Management of Cohn's Disease in Adults. Amer J Gastroenterol 113: 481-517. 